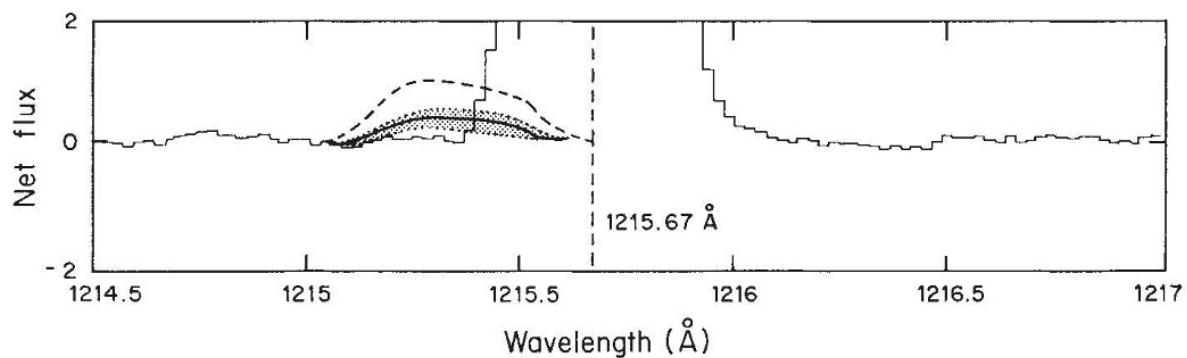

FIG. 2 Observed IUE spectrum near 1,216 Å with $2.5 \mathrm{kR}$ of $D$ Ly- $\alpha$ (dashed line) and $1.0 \pm 0.4 \mathrm{kR}$ (stippled region).

optical depth of the corresponding deuterium distribution being reduced. A significant concentration of hydrogen in the form of compounds (such as $\mathrm{H}_{2}$ and $\mathrm{HCl}$ ) at $110 \mathrm{~km}$ could cause such a change in the hydrogen altitude profile. Although ionospheric physics requires the mass- 2 species to be mostly $\mathrm{D}$ and excludes 10 p.p.m. of $\mathrm{H}_{2}$ at $100 \mathrm{~km}$, it may allow 1 or 2 p.p.m. of $\mathrm{H}_{2}$.

Note that the figure in the Bertaux and Clarke letter is misleading and its caption is incorrect in asserting that a $\mathrm{D} / \mathrm{H}$ ratio of $1.6 \times 10^{-2}$ would lead to $2.5 \mathrm{kR}$ of D Ly- $\alpha$. The authors mistakenly take the upward flux of hydrogen in Paxton's analysis $\left(7.5 \times 10^{7} \mathrm{~cm}^{-2} \mathrm{~s}^{-1}\right)$ to be escape flux. Almost all this upward flux goes to support global circulation. Hence it is appropriate to treat deuterium in a onedimensional model as carrying an analogous flux. Proper calculation of the deuterium column density gives $(3 \pm 1.2)$ $\times 10^{11} \mathrm{~cm}^{-2}$ when the $\mathrm{D} / \mathrm{H}$ ratio at $100 \mathrm{~km}$ is $(1.6 \pm 0.2) \times 10^{-2}$. The D Ly- $\alpha$ emission rate would then be $(1.0 \pm 0.4) \mathrm{kR}$, not $2.5 \mathrm{kR}$ (Fig. 2). $300 \mathrm{R}$ of D Ly- $\alpha$ would imply a $\mathrm{D} / \mathrm{H}$ ratio of $(5.0 \pm 2.1) \times 10^{-3}$ at $100 \mathrm{~km}$ in the Paxton et al. hydrogen model.

THOMAS M. DONAHUE

Department of Atmospheric, Oceanic and Space Sciences,

Space Physics Research Laboratory,

University of Michigan,

Ann Arbor, Michigan 48109-2143, USA

1. Bertaux, J.-L. \& Clarke, J.T. Nature 338, 567-568 (1989).

2. Paxton, L.J., Anderson, D.E. Jr \& Stewart, A.I.F. J. geophys. Res. 93, 1766-1772 (1988).

3. McElroy, M.B., Prather, M.J. \& Rodriguez, J.M. Science 215, 1614 (1982).

4. Hartle, R.E. \& Taylor, H.A. Jr Geophys. Res. Lett. 10, 965 (1983).

5. Kumar, S. \& Taylor, H.A. Jr Icarus 62, 494-504 (1985).

6. Donahue, T.M., Hoffman, J.H., Hodges, R.R. Jr \& Watson A.J. Science 216, 630-633 (1982).

7. Kumar, S., Hunten, D.M. \& Taylor, H.A. Jr Geophys. Res. Lett. 8, 237 (1981).

Bertaux and Clarke reply-All the arguments presented by Donahue are sensible, but each allows room for discussion. Rather than going into the details here, we suggest another test of the presence of $\mathrm{D}$ atoms in the upper atmosphere of Venus. These atoms should produce an important limb brightening, just over the altitude at which $\mathrm{CO}_{2}$ becomes transparent (horizontally) to Ly- $\alpha$ radiation, at about $125-130 \mathrm{~km}$ of altitude. This is because the D atmosphere is optically thin, whereas $\mathrm{H}$ atmosphere is not. With a
$\mathrm{D} / \mathrm{H}$ ratio of $1.6 \times 10^{-2}$ and the $\mathrm{H}$ model proposed by Donahue, this $\mathrm{D}$ Ly- $\alpha$ brightening should amount to 1-3 kR, superimposed on the smoother $\mathrm{H} \mathrm{Ly}-\alpha$ brightening. Perhaps the data collected by the ultraviolet spectrometer on board Pioneer Venus at low altitude could be re-examined carefully for the detection of such a deuterium limb brightening.

Service d'Aéronomie du CNRS J.-L. BertauX BP3, 91371 Verriéres le Buisson, France J. T. CLARKE

Department of Atmospheric, Oceanic and Space Sciences,

University of Michigan,

Ann Arbor, Michigan 48109, USA

\section{Heart disease risks}

SIR-Barker writes that "the geographical distribution of ischaemic heart disease is closely related to that of poor child health and development, indicated by high infant and child mortality about 70 years ago"1. According to this aetiological model, people born in a relatively poor area, who subsequently move to a more affluent part of Britain will experience a higher incidence of ischaemic heart disease (IHD) than those who have always lived in the more affluent area. Conversely, regardless of where they eventually settle, people born and raised in more affluent areas will suffer less adult heart disease. Migration into, and within, Britain, provides the opportunity to test the hypothesis.

The British Regional Heart Study reported $^{2}$ a strong geographical gradient in the risk of a major IHD event in 7,735 middle-aged men, living in 24 towns in England, Scotland and Wales, who were followed up between 1978 and 1986. Regardless of where they were born, men examined in Scotland experienced the highest IHD risk, whereas those examined in the south of England had the lowest. Men who were born in the relatively poor part of Britain, north of a line drawn between the Severn estuary and the Wash, but who later moved to the comparatively affluent south, experienced a risk of IHD little different from men who had always lived south of the line. On the other hand, men born in the south who moved north experienced a higher risk than those who remained in the south. Those who moved north faced an IHD risk similar to that of men who had always lived there. Furthermore, immigrants to Britain also adopted the IHD risk of the part of the country to which they moved, regardless of their birthplace.

These findings are consistent with other epidemiological studies of migrants. Between 1962 and 1966, immigrants to Australia from Italy, England and Wales had lower death rates from cardiovascular disease than Australian-born women and men $^{3}$, but death rates rose progressively with increasing length of residence. Also, between 1970 and 1972 immigrants into England and Wales had, in general, IHD mortality rates intermediate between those of their original and new country of residence ${ }^{4}$.

Thus, environmental and cultural factors acting in adult life are of crucial importance in the development of coronary heart disease, independent of birthplace. It seems unlikely that geographical differences in IHD risk can be substantially explained by the prenatal and childhood environment.

JONATHAN ELFORD A.G. SHAPER

Department of Clinical Epidemiology and General Practice,

Royal Free Hospital School of Medicine, London NW3 2PF, UK

1. Barker, D.J.P. Nature 338, 371-372 (1989)

2. Elford, J. et al. Lancet I, 343-346 (1989)

. Stenhouse, N.S. \& McCall, M.G. J. chron. Dis. 23, 423 (1970).

4. Marmot, M.G. et al. Immigrant Mortality in England and Wales, 1970-78 (HMSO, London, 1984).

\section{Spin glass in a whirl}

SIR-Sourlas's use of spin-glass models as error-correcting codes (Nature 339, 693; 1989 ) is a complex way of saying something essentially very simple: that binary arithmetic, as used in most conventional computers, is multiply connected. This is in contradistinction to Gray, or reflected, binary code, which is simply connected. This means, for example, that in conventional computers five switching operations, (binary 01111 to 10000 ), are required in changing from the binary code for $15_{10}$ to the code for $16_{10}$; there are thus factorial five $\left(120_{10}\right)$ possible switching sequences. This fundamental flaw in conventional computation has been drawn to the attention of the UK Institution of Electrical Engineers in relation to the preparation of software intended for use in safety-critical situations, and also to the attention of the Combined Higher Education Software Team.

15 Everest Drive,

B. E. P. Clement

Crickhowell,

Powys NP8 1DH, UK 